Check for updates

Cite this: Mater. Horiz., 2019, 6, 1512

Received 21st February 2019, Accepted 17th April 2019

DOI: $10.1039 / \mathrm{c} 9 \mathrm{mh} 00279 \mathrm{k}$

rsc.li/materials-horizons

\section{Magnetically driven piezoelectric soft microswimmers for neuron-like cell delivery and neuronal differentiation $\dagger$}

\author{
Xiang-Zhong Chen, (D) $\ddagger^{\mathrm{a}}$ Jia-Hao Liu, (D) $\ddagger^{\mathrm{b}}$ Mei Dong, $\ddagger^{\mathrm{b}}$ Lucas Müller, ${ }^{\mathrm{a}}$ \\ George Chatzipirpiridis, ${ }^{a}$ Chengzhi Hu, (D $\S^{a}$ Anastasia Terzopoulou, (D) ${ }^{a}$ \\ Harun Torlakcik, ${ }^{a}$ Xiaopu Wang, (D) a Fajer Mushtaq, ${ }^{a}$ Josep Puigmartí-Luis, (D) c \\ Qun-Dong Shen, iD bradley J. Nelson ${ }^{a}$ and Salvador Pané*a
}

\begin{abstract}
Wireless micro- and nanorobots are biomedical devices with a potential use in high-precision minimally invasive therapies. Here, a highly integrated multifunctional soft microrobot is developed for targeted cell therapy applications, featuring targeted cell transportation and induced cell differentiation. The micromachines are made of composites consisting of a soft piezoelectric polymer matrix in which magnetic nanoparticles are dispersed. The magnetic nanoparticles serve as the component for the device's magnetic actuation, while the piezoelectric polymer acts as both a steerable scaffold and an acoustically responsive cell electrostimulation platform. With the application of a rotating magnetic field, the microrobots swim in a corkscrew motion in different liquid environments that mimic human body fluids. When the swimmers are subjected to acoustic waves, their piezoelectric body is electrically polarized which induces cell differentiation of neuron-like PC12 cells loaded on the swimmers surface. This combinatorial technique may open up new avenues for bioelectronic therapies.
\end{abstract}

Traumatic injury and neurodegenerative diseases in the central nervous system, such as Alzheimer's and Parkinson's, result in irreversible structural destruction of the neuronal network accompanied by neuronal damage or apoptosis. ${ }^{1,2}$ Unfortunately,

\footnotetext{
${ }^{a}$ Multi-Scale Robotics Lab (MSRL), Institute of Robotics \& Intelligent Systems (IRIS), ETH Zurich, Zurich 8092, Switzerland. E-mail: vidalp@ethz.ch

${ }^{b}$ Department of Polymer Science and Engineering and Key Laboratory of High Performance Polymer Materials and Technology of MOE, School of Chemistry and Chemical Engineering, Nanjing University, Nanjing 210023, China

${ }^{c}$ Institute of Chemical and Bioengineering, ETH Zurich, Vladimir Prelog Weg 1, 8093 Zurich, Switzerland

$\dagger$ Electronic supplementary information (ESI) available: Experimental section, parameter control of the helical microswimmer, frequency-dependent swimming behaviour of the helical micro swimmer in DI water, optimization of swimming properties by optimizing the parameter, the differentiation of the cells at day 3 . See DOI: $10.1039 / \mathrm{c} 9 \mathrm{mh} 00279 \mathrm{k}$

$\$$ Authors contributed equally.

$\S$ Present address: Department of Mechanical and Energy Engineering, Southern University of Science and Technology, No. 1088, Xueyuan Blvd., Xili, Nanshan District, Shenzhen, Guangdong, China.
}

\section{New concepts}

Neurons in the adult human brain have a limited capability for regeneration, which is one of the major hurdles in the cure of neuronal traumatic injuries and neuron degenerative diseases, however neurogenesis can still occur in a favorable or stimulated environment. The delivery of neural progenitor cells to a targeted area, and how to effectively induce their differentiation, are the major challenges to be overcome. The use of remotely controlled micro- and nanorobots shows potential for future high-precision minimally invasive therapies. In this paper, we proposed a conceptual platform that is able to transport cells in a targeted and precise way, while creating an electrically stimulated microenvironment to favor their differentiation under ultrasonic vibration. This work presents a unique concept that could inspire minimally invasive cell therapies for treatment of neuronal injury or diseases.

neurons in the adult human brain have a limited regeneration capability. ${ }^{3}$ Cell therapy shows great potential to repair and regenerate damaged or diseased tissues. Stem cells or neural progenitor cells can be directed to differentiate into neurons, replace the damaged neural tissues and form functional neuronal networks. ${ }^{4}$ However, the blood-brain barrier (BBB) hinders effective recruitment of the therapeutic cells to specific sites of the central nervous system (CNS) if they are intravenously administered. Therefore, cell transplantation to tissues of the central nervous system usually requires conventional medical or surgical interventions, which are not only risky and time-consuming, but also painful for the patient. An injection of therapeutic agents into the subarachnoid space (also known as intrathecal administration) could be an option to circumvent the BBB. The subarachnoid space is the volume between two meningeal membranes, the arachnoid membrane and the pia mater, and is filled with cerebrospinal fluid (CSF). Although it has recently been demonstrated that intrathecally injected stem cells loaded with superparamagnetic nanoparticles can be steered using permanent magnets, ${ }^{5}$ the targeted delivery of therapeutic cells and their differentiation at specific sites of the central nervous system remains challenging.

In this work, we demonstrate that neuron-like cells can be transported to a targeted area and can be induced to differentiate 
by magnetically driven piezoelectric helical microswimmers. Untethered small-scale robotic devices are potential tools for biomedical applications such as targeted therapy delivery and minimally invasive interventions. ${ }^{6-17}$ The use of magnetic fields is a popular method for maneuvering micro- and nanorobots for biomedical applications, as magnetic energy is harmless to cells and tissues in a wide range of conditions, and can also penetrate deeply into the human body. ${ }^{18,19}$ While several magnetic microand nanomachine designs allow for the transportation of cells to target areas, most do not provide a stimulating environment for the cells to proliferate or differentiate, which is crucial for targeted cell therapy, especially in the area of neural diseases. A very recent contribution illustrates the importance of designing highly integrated microtransporters with bioactive features. Particularly, Yasa et al. have shown that magnetically driven robotic microtransporters incorporating both stem cells and stem cell niche mimetic microenvironments can be used to successfully program the differentiation of bone cells. ${ }^{20}$ For neuronal cells, it has been demonstrated that the deficiency of neuronal regeneration is not an intrinsic cell property, but is rather caused by an unfavorable growth environment within the damaged tissue. ${ }^{21}$ Under external strain such as ultrasonic vibration, piezoelectric materials can provide electrical stimulation to promote neuron differentiation without nerve growth factors (NGF). ${ }^{22-27}$ Therefore, in this work, we designed microswimmers made of helical bodies consisting of a piezoelectric polymer matrix dispersed with magnetic nanoparticles. The magnetic nanoparticles serve as the motile component of the device, while the piezoelectric polymer acts as both a steerable scaffold and an acoustically responsive cell electrostimulation platform.

Fig. 1a depicts the fabrication process of the piezoelectric magnetic microswimmer. First, magnetic nanoparticles were mixed in a piezoelectric polymer solution to obtain a uniform dispersion. The choice of the piezoelectric material is critical, as it is essential for inducing cell differentiation. Piezoelectric polymers, such as polyvinylidene fluoride-co-trifluoroethylene (P(VDF-TrFE)) and poly-L-lactic acid (PLLA), are particularly promising due to their easy-processing, mechanical robustness, flexibility, chemical stability, and biocompatibility. ${ }^{26,28} \mathrm{CoFe}_{2} \mathrm{O}_{4}$ (CFO) was selected as the magnetic component due to its hardmagnetic nature, which enables the programming of the magnetization direction of the composite structure. The remanence magnetization of CFO allows the composite helical structure to be permanently magnetized perpendicular to its long axis. In this way, the helix can rotate along its short axis when it is actuated with a rotating magnetic field, causing it to swim with a corkscrew motion. The polymer/nanoparticle dispersion was applied to a copper wire through dip-coating to form a uniform composite layer after the solvent evaporated. The coated wire was then fixed onto a custom-made sample holder (Fig. S1, ESI $\dagger$ ) and a laser mill was used to ablate the composite film. The laser beam was moved along the copper wire while the copper wire was rotating around its short axis at a certain speed, so that the trajectory of the laser ablation on the cylindrical surface formed a helical shape. Finally, the helical microswimmer was released by selectively etching the copper wire. The detailed
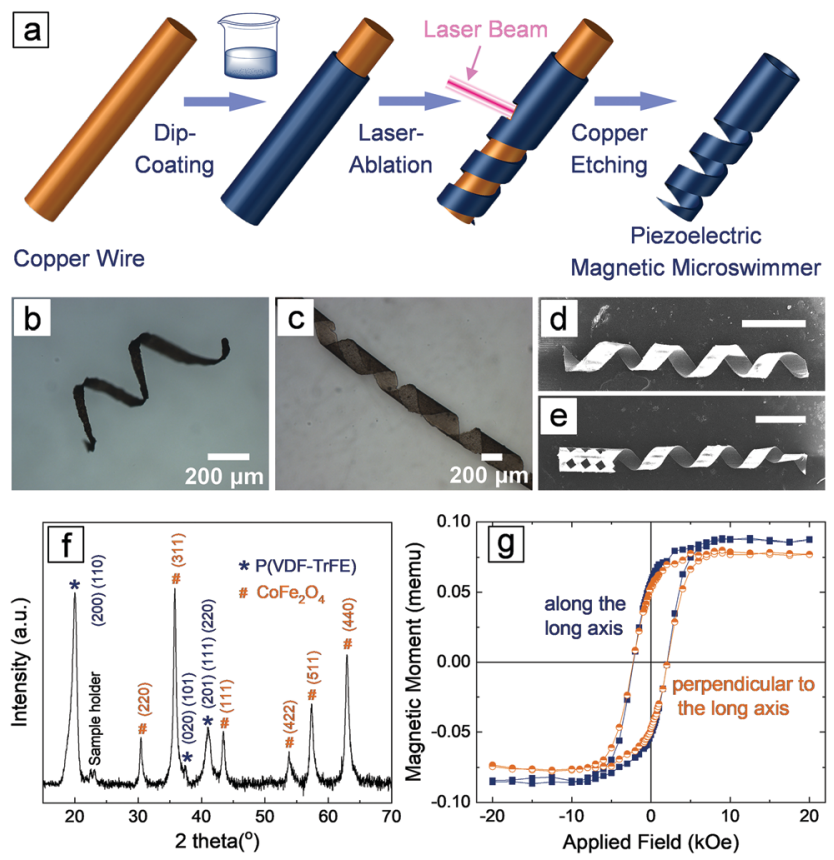

Fig. 1 (a) Schematic illustration showing the fabrication of piezoelectric magnetic microswimmers. ( $b$ and c) Optical images of two types of helical microswimmers with different pitches and etch ratios. (d and e) SEM images of helical microswimmers without a head (d) and with a head (e). The scale bars in ( $d$ and e) represent $400 \mu \mathrm{m}$. (f) XRD pattern of P(VDFTrFE)/CFO composite film. (g) The magnetic hysteresis loop of one helical magnetic microswimmer.

fabrication process can be found in the experimental and ESI $\dagger$ sections. Note that the current fabrication method allows for easy tuning of the parameters of the helical structures. The pitch, pitch angle and the ratio of the etched composite materials can be controlled by tuning the speed of the laser beam motion, the rotating speed of the wire and the laser spot size (see ESI $\dagger$ ). This approach is not limited to the current composite system, but is applicable to other polymers and composites. Fig. 1b shows a helical microswimmer made of PLLA/CFO with a pitch angle of $25^{\circ}$, inner diameter of $250 \mu \mathrm{m}$ and etching ratio of $70 \%$. The $\mathrm{P}(\mathrm{VDF}-\mathrm{TrFE}) / \mathrm{CFO}$ helical swimmer in Fig. 1c has a pitch angle of $35^{\circ}$, inner diameter of $250 \mu \mathrm{m}$, and etching ratio of $30 \%$. Fig. 1d and e show two helical swimmers with identical diameters, pitch angles, and etching ratios, one of which has a carved head.

The addition of nanoparticles into polymer matrices may result in changes in the processability of the composite, and the interaction between nanoparticles and polymers may induce significant changes in the crystalline structures of the piezoelectric polymer. ${ }^{29,30}$ To confirm that $\mathrm{P}(\mathrm{VDF}-\mathrm{TrFE})$ maintains its piezoelectric phase, and that the CFO particles were not deteriorated by the processing procedure (e.g., ultrasonic assisted dispersion, heat generated by the laser, etc.), we performed X-ray diffraction (XRD) measurements on the P(VDF-TrFE)/CFO composite (Fig. 1f). The XRD of the composite reveals that $\mathrm{P}(\mathrm{VDF}-\mathrm{TrFE})$ is still piezoelectric as evidenced by the diffraction peak at $20.0^{\circ}$, which reflects the $(110,200)$ crystallographic plane with an interchain lattice space of $4.43 \AA$ in ferroelectric $\beta$ phase. ${ }^{31-33}$ The diffraction peaks located 
at $30.4^{\circ}, 35.8^{\circ}, 43.4^{\circ}, 53.8^{\circ}, 57.4^{\circ}$, and $62.9^{\circ}$ represent the $(220)$, (311), (111), (422), (511) and (440) crystallographic planes of CFO (JSPDS: No. 22-1086), respectively. The magnetic properties were examined using a vibrating sample magnetometer (VSM), and the representative curves of a single helical composite structure are shown in Fig. 1g. The paramagnetic contribution was subtracted to reveal the ferromagnetic nature of the composite. The diagram shows that the coercive magnetic fields are almost the same $(\sim 2.1 \mathrm{kOe})$ both along, and perpendicular to, the long axis of the helical swimmers. The remnant magnetization values for both directions are also similar, 0.0547 memu along the long axis and 0.0504 memu perpendicular to the long axis, respectively. No obvious magnetic anisotropy, i.e., no preferred magnetization easy axis, was observed in the helical structure.

Magnetic propulsion shows great potential for the locomotion of micro- and nanorobots in the human body because it is biocompatible and noninvasive..$^{7,14,34-36}$ In order to actuate a small-scale magnetic robot, a magnetic field, either inhomogeneous (magnetic field gradient) or time-varying (rotating or oscillating), must be applied. Magnetic helical structures are able to transform their rotational motion into translational motion even under low magnitude rotating magnetic fields. The corkscrew locomotion mechanism is one of the most efficient propulsion strategies at low Reynold number regime, where viscous forces dominate over initial forces. ${ }^{36,37}$ The helical structures were magnetized along their short axis using a strong static magnetic field generated by an NdFeB permanent magnet to actuate rotation around their short axis. When the magnetic field was removed, a remanent magnetization $M_{\mathrm{r}}$ remained. When a rotating magnetic field, $H$, is applied, the microswimmer rotates because $M_{\mathrm{r}}$ tends to align itself with the direction of $H$, as is shown in Fig. 2a. In Fig. 2b, a rotating magnetic field of $10 \mathrm{mT}$ was applied to control the motion of the microswimmer in deionized (DI) water. The corresponding video can be found in the $\mathrm{ESI} \dagger$ (Video SV1). The total velocity of the microswimmer is composed of two parts: forward velocity and lateral velocity, as schematically illustrated. The lateral velocity was generated by friction with the substrate surface and the rolling motion of the helical microswimmer. ${ }^{36}$ The swimming velocities, both forward and lateral, and the drift angle (which is defined as the angle between the forward and lateral velocity direction) are dependent on the rotating frequency of the applied magnetic field (Table S1, ESI $\dagger$ ), which can also be seen in other works. ${ }^{38}$ Our aim is to inject the microswimmer in a minimally invasive way, for example by lumbar puncture, actuating the microswimmer in the subarachnoid space with magnetic fields. The subarachnoidal cavity in the spinal cord is a narrow space filled with the cerebrospinal fluid (CSF), which has a viscosity very close to water. We used a capillary tube filled with DI water to simulate the magnetically controlled movement of the microswimmer in the subarachnoidal cavity, as shown in Fig. 2c and Video SV2 (ESI $\dagger$ ). Confined by the tube wall, the microswimmer can only move forwards or backwards along the tube, and is controlled by changing the rotating direction (clockwise or counter-clockwise), which allows the microswimmer to be retrieved after completion of its delivery task. The speed is linearly proportional to the frequency of the

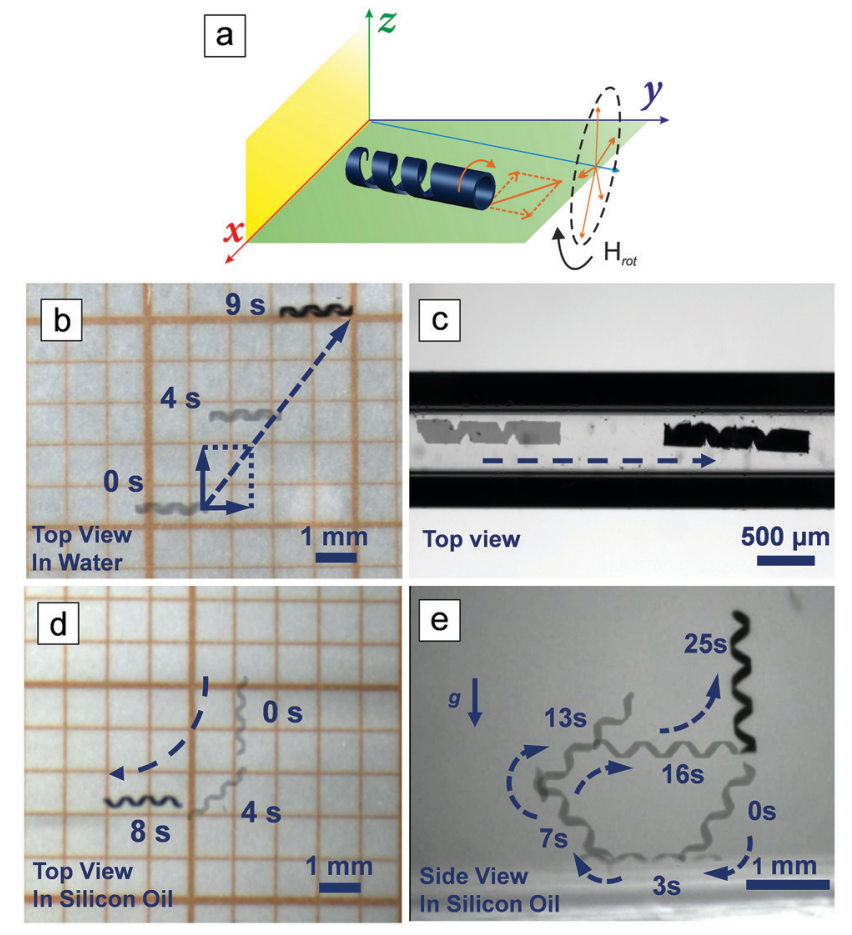

Fig. 2 (a) Schematic illustration of the microswimmer's motion in water under a rotating magnetic field. The time-lapse images of a micro helical swimmer actuated under a rotating magnetic field on a plenary surface in water (b) and in a narrow capillary tube filled with water (c). The swimming capability of the micro swimmer in 3D in viscous environment is demonstrated in silicon oil from a top view (d) and side view (e).

rotating magnetic field when the frequency is lower than $15 \mathrm{~Hz}$ (Fig. S2, ESI $\dagger$ ). No obvious step-out behavior was observed in the assessed frequency range, although at $30 \mathrm{~Hz}$ the speed is slightly less than the predicted value, and a speed of $\sim 580 \mu \mathrm{m} \mathrm{s}^{-1}$ was achieved.

We also need to consider the motion of the microswimmer once it reaches the brain. It is reasonable to assume that the helical microswimmers will need to swim through biological micro-environments, such as the viscous extracellular matrix (ECM), to deliver the cells to a targeted area. The biological environment of the ECM is a highly complex medium composed of many types of macromolecules, with a viscosity that can exceed $200 \mathrm{mPa} \mathrm{S},{ }^{39}$ which is much higher than that of water and $\mathrm{CSF}(\sim 1 \mathrm{mPa}$ ). The Reynolds number is defined as

$$
\operatorname{Re}=u L / \nu
$$

where $u$ is the velocity of the fluid with respect to the object $\left(\mathrm{m} \mathrm{s}^{-1}\right), L$ is a characteristic linear dimension $(\mathrm{m})$, and $\nu$ is the kinematic viscosity of the fluid $\left(\mathrm{m}^{2} \mathrm{~s}^{-1}\right)$. Considering $u$ as $200 \mu \mathrm{m} \mathrm{s}^{-1}, L$ as $2000 \mu \mathrm{m}$, and $\nu$ as $200 \mathrm{~mm}^{2} \mathrm{~s}^{-1}$, the Reynolds number will be in the order of $10^{-3}$. The corkscrew motion of the microswimmer is advantageous in such a low Reynolds number environment. In order to simulate motion in these conditions, we actuated the micro helical swimmer in silicon oil, which has a viscosity of around $340 \mathrm{mPa}$ S. The helical structure should be optimized in terms of its pitch angle, length and etch ratio, as discussed in the $\operatorname{ESI} \dagger$ (Section 4). 
Fig. 2d and Video SV3 (ESI $\dagger$ ) show how one microswimmer with optimized parameters can be actuated to swim in silicon oil at a frequency of $5 \mathrm{~Hz}$, with a swimming speed of up to $0.5 \mathrm{~mm} \mathrm{~s}^{-1}$. Fig. 2e and Video SV4 (ESI $\dagger$ ) show the side view of the microhelical swimmer being actuated to swim in silicon oil. The swimmer can be actuated to swim precisely in 3D, even against gravity, and this precise locomotion in viscous fluid shows the potential of microswimmers for use in biological environments. Fig. 1e shows a microswimmer with a head, which was also actuated to swim in silicon oil in $3 \mathrm{D}$, however the maximum swimming frequency achieved was $1.8 \mathrm{~Hz}$ (Video SV5, ESI $\dagger$ ).

To deliver cells to the targeted area and stimulate them to differentiate in situ, the microswimmer must be able to accommodate the cells on their structure, as shown in Fig. 3a. PC12 cells were selected, as this cell line has been widely used as a model for neuronal differentiation. The microswimmers were
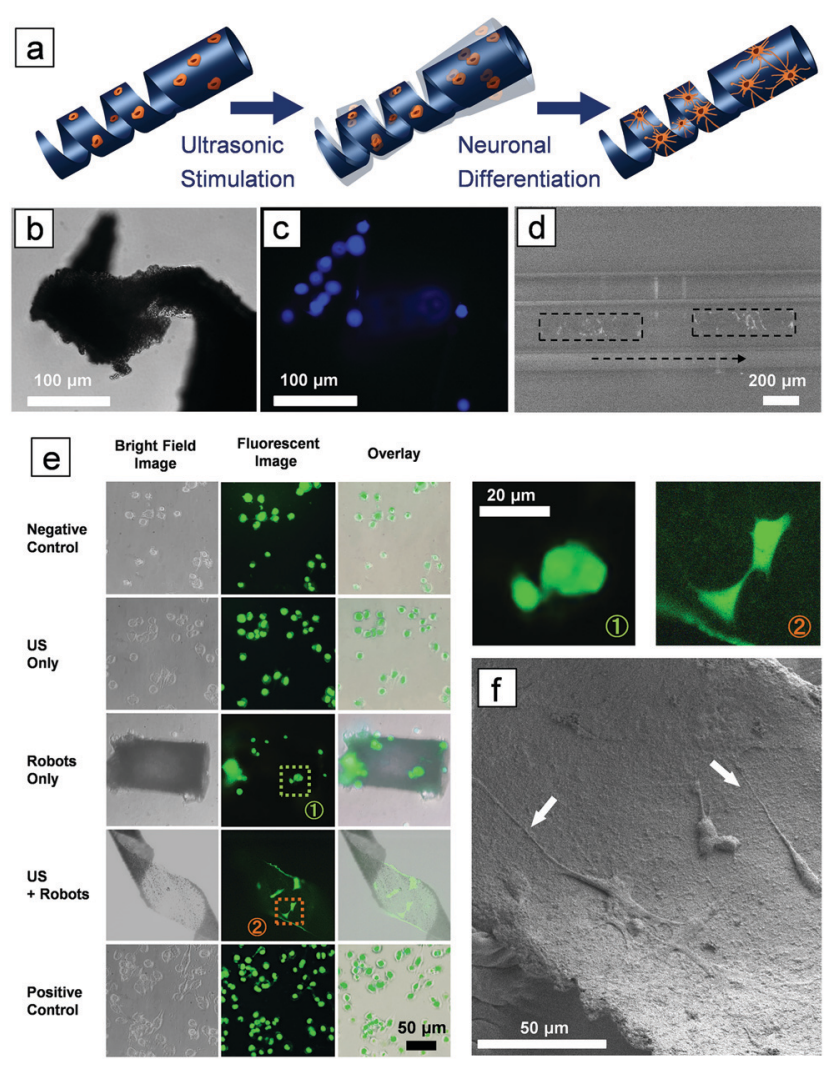

Fig. 3 (a) Schematic illustration: ultrasound (US) stimulation of the piezoelectric microswimmer induces neuronal differentiation of PC12 cells (b) Bright-field and (c) fluorescent microscopic image of a tail of a helical microswimmer loaded with PC12 cells. (d) A time-lapse fluorescent image of a cell-laden micro helical swimmer actuated under a rotating magnetic field in a capillary tube filled with water. The boxes indicate the position of the microswimmer at two time points. The light spots on the microswimmers are the cells whose nuclei were stained by Hoechst. (e) Comparison of PC12 cells cultured in media (negative control), media with ultrasonic stimulation (US only), cultured on microrobots without ultrasonic stimulation (robots only), cultured on microrobots with ultrasonic stimulation (US + robots), and cultured with NGF (positive control). The enlarged images on the right show in more detail the two highlighted cells on the middle left. (f) An SEM image showing differentiated PC12 cells on the inner surface of a helical swimmer after US stimulation. treated with poly-L-lysine, a synthetic amino acid chain, to facilitate the adhesion of cells to their surface. Fig. 3b shows an optical image of a microswimmer tail made of PLLA and magnetic particles, Fig. $3 \mathrm{c}$ is the corresponding fluorescent image, which shows PC12 cells attached to the microswimmer. The blue dots in the figure are cell nuclei stained with Hoechst. As the microswimmer is not transparent, only the cells attached at the edges of the helical body are visible. The cell-laden microswimmer can then be actuated to move in narrow spaces, as shown in Fig. $3 \mathrm{~d}$ and in Video SV6 (ESI $\dagger$ ), which were taken under an inverted fluorescent microscope, where only the cells on the edges can be visualized. After the microswimmer reaches the target area, the cells can be induced to differentiate using ultrasound stimulation. After 3 days, PC12 cells incubated on the P(VDF-TrFE)/magnetic nanoparticle microswimmers began to form protrusions under acoustic stimulation (Fig. S4, ESI $\dagger$ ). Fig. 3e shows that after 7 days the PC 12 cells are already differentiated, while the control groups seeded on PVDF microswimmers without ultrasound stimulation, and those only stimulated with ultrasound in the absence of microswimmers, remain undifferentiated. The differentiated PC12 cells can be clearly distinguished from the undifferentiated cells by examining the extended axons, as observed from the enlarged fluorescent images on the right panel of Fig. 3e. Fig. $3 f$ is an SEM image showing differentiated PC12 cells on the inner surface of a PVDF helical swimmer after 7 days of US stimulation. The axons are clearly observed, as indicated by the white arrows.

Regeneration of neuron cells in an adult brain is a key step in the construction of a functional neuronal network. In this work, we propose a conceptual methodology for contactless, controlled neuron cell delivery and in situ differentiation for neuroregenerative therapy. By combining the magnetically controlled microswimmer and piezoelectric material, we fabricated a mobile platform for delivery and stimulation of neuron-like cells. Remarkably, the microswimmers developed in our work could actively induce the differentiation of neuronal cells only under physical stimulation, circumventing the use of expensive growth factors. The design and usage of microswimmers with other geometries would also be possible, assuming they could be actuated to deliver cells to targeted regions. The external energy sources for both actuation (magnetic fields) and stimulation (ultrasound) are biocompatible and can penetrate deeply into the human body. Related techniques have already been widely adopted in medical imaging procedures such as magnetic resonance imaging (MRI) and ultrasound-based imaging. However, the in vivo application of these microagents will require further refinements and optimization. Note that the long-term biosafety of piezoelectric and magnetic compounds, their biodegradability and the physiological management of these materials by the body environments will require a further in-depth assessment. Nevertheless, we anticipate the present concept could provide inspiration for treating traumatic neuron injuries and neuron degenerative diseases.

\section{Conflicts of interest}

There are no conflicts to declare. 


\section{Acknowledgements}

This work has been financed by the European Research Council Starting Grant "Magnetoelectric Chemonanorobotics for Chemical and Biomedical Applications (ELECTROCHEMBOTS) under the grant no. 336456. The authors would like to acknowledge Lydia Zehnder from Institute für Geochemie und Petrologie of ETH for her kind support on XRD measurements, the Scientific Center for Optical and Electron Microscopy (ScopeM) of ETH and the FIRST laboratory for their technical support.

\section{Notes and references}

1 A. A. Farooqui, Phytochemicals, Signal Transduction, and Neurological Disorders, Springer, New York, Heidelberg, London, 1st edn, 2012.

2 E. Radi, P. Formichi, C. Battisti and A. Federico, J. Alzheimer's Dis., 2014, 42(Suppl 3), S125-S152.

3 M. M. Steward, A. Sridhar and J. S. Meyer, Curr. Top. Microbiol. Immunol., 2013, 367, 163-191.

4 O. Lindvall and Z. Kokaia, J. Clin. Invest., 2010, 120, 29-40. 5 D. Tukmachev, O. Lunov, V. Zablotskii, A. Dejneka, M. Babic, E. Sykova and S. Kubinova, Nanoscale, 2015, 7, 3954-3958.

6 H. Ceylan, J. Giltinan, K. Kozielski and M. Sitti, Lab Chip, 2017, 17, 1705-1724.

7 J. Li, B. Esteban-Fernández de Ávila, W. Gao, L. Zhang and J. Wang, Sci. Robot., 2017, 2, eaam6431.

8 D. Walker, B. T. Kasdorf, H. H. Jeong, O. Lieleg and P. Fischer, Sci. Adv., 2015, 1, e1500501.

9 H. Wang and M. Pumera, Chem. Rev., 2015, 115, 8704-8735.

10 L. Zheng, L.-g. Chen, H.-b. Huang, X.-p. Li and L.-l. Zhang, Microsyst. Technol., 2016, 22, 2371-2387.

11 J. Katuri, X. Ma, M. M. Stanton and S. Sánchez, Acc. Chem. Res., 2017, 50, 2-11.

12 M. Hoop, Y. Shen, X. Z. Chen, F. Mushtaq, M. S. Sakar, A. Petruska, M. J. Loessner, B. J. Nelson and S. Pané, Adv. Funct. Mater., 2016, 26, 1063-1069.

13 S. Kim, F. Qiu, S. Kim, A. Ghanbari, C. Moon, L. Zhang, B. J. Nelson and H. Choi, Adv. Mater., 2013, 25, 5863-5868.

14 B. J. Nelson, I. K. Kaliakatsos and J. J. Abbott, in Annual Review of Biomedical Engineering, ed. M. L. Yarmush, J. S. Duncan and M. L. Gray, 2010, vol. 12, pp. 55-85.

15 M. Medina-Sanchez and O. G. Schmidt, Nature, 2017, 545, 406-408.

16 M. Medina-Sanchez, L. Schwarz, A. K. Meyer, F. Hebenstreit and O. G. Schmidt, Nano Lett., 2016, 16, 555-561.

17 X.-Z. Chen, M. Hoop, N. Shamsudhin, T. Huang, B. Ozkale, Q. Li, E. Siringil, F. Mushtaq, L. Di Tizio, B. J. Nelson and S. Pané, Adv. Mater., 2017, 29, 1605458.

18 X. Z. Chen, N. Shamsudhin, M. Hoop, R. Pieters, E. Siringil, M. S. Sakar, B. J. Nelson and S. Pané, Mater. Horiz., 2016, 3, 113-118.
19 X.-Z. Chen, M. Hoop, F. Mushtaq, E. Siringil, C. Hu, B. J. Nelson and S. Pané, Appl. Mater. Today, 2017, 9, 37-48.

20 I. C. Yasa, A. F. Tabak, O. Yasa, H. Ceylan and M. Sitti, Adv. Funct. Mater., 2019, 29, 1808992.

21 P. M. Richardson, U. M. Mcguinness and A. J. Aguayo, Nature, 1980, 284, 264-265.

22 M. Hoop, X.-Z. Chen, A. Ferrari, F. Mushtaq, G. Ghazaryan, T. Tervoort, D. Poulikakos, B. Nelson and S. Pané, Sci. Rep., 2017, 7, 4028.

23 G. Ciofani, S. Danti, D. D’Alessandro, L. Ricotti, S. Moscato, G. Bertoni, A. Falqui, S. Berrettini, M. Petrini, V. Mattoli and A. Menciassi, ACS Nano, 2010, 4, 6267-6277.

24 A. Marino, S. Arai, Y. Y. Hou, E. Sinibaldi, M. Pellegrino, Y. T. Chang, B. Mazzolai, V. Mattoli, M. Suzuki and G. Ciofani, ACS Nano, 2015, 9, 7678-7689.

25 A. H. Rajabi, M. Jaffe and T. L. Arinzeh, Acta Biomater., 2015, 24, 12-23.

26 C. Ribeiro, V. Sencadas, D. M. Correia and S. LancerosMendez, Colloids Surf., B, 2015, 136, 46-55.

27 G. G. Genchi, L. Ceseracciu, A. Marino, M. Labardi, S. Marras, F. Pignatelli, L. Bruschini, V. Mattoli and G. Ciofani, Adv. Healthcare Mater., 2016, 5, 1808-1820.

28 X. Chen, S. Pan, P. J. Feng, H. Bian, X. Han, J. H. Liu, X. Guo, D. Chen, H. Ge and Q. D. Shen, Adv. Mater., 2016, 28, 10684-10691.

29 X.-Z. Chen, X. Li, X.-S. Qian, M. Lin, S. Wu, Q.-D. Shen and Q. Zhang, Polymer, 2013, 54, 5299-5302.

30 B. Chu, M. Lin, B. Neese, X. Zhou, Q. Chen and Q. M. Zhang, Appl. Phys. Lett., 2007, 91, 122909.

31 W. Xia, Z. Xu, F. Wen, W. Li and Z. Zhang, Appl. Phys. Lett., 2010, 97, 222905.

32 X.-Z. Chen, X. Li, X.-S. Qian, S. Wu, S.-G. Lu, H. Gu, M. Lin, Q.-D. Shen and Q. Zhang, Polymer, 2013, 54, 2373-2381.

33 X.-Z. Chen, X. Chen, X. Guo, Y.-S. Cui, Q.-D. Shen and H.-X. Ge, Nanoscale, 2014, 6, 13945-13951.

34 X. Yan, Q. Zhou, M. Vincent, Y. Deng, J. Yu, J. Xu, T. Xu, T. Tang, L. Bian, Y.-X. J. Wang, K. Kostarelos and L. Zhang, Sci. Robot., 2017, 2, eaaq1155.

35 X.-Z. Chen, B. Jang, D. Ahmed, C. Hu, C. De Marco, M. Hoop, F. Mushtaq, B. J. Nelson and S. Pane, Adv. Mater., 2018, 30, 1705061.

36 K. E. Peyer, L. Zhang and B. J. Nelson, Nanoscale, 2013, 5, 1259-1272.

37 J. J. Abbott, K. E. Peyer, M. C. Lagomarsino, L. Zhang, L. Dong, I. K. Kaliakatsos and B. J. Nelson, Int. J. Robot. Res., 2009, 28, 1434-1447.

38 X. Wang, X.-H. Qin, C. Hu, A. Terzopoulou, X.-Z. Chen, T.-Y. Huang, K. Maniura-Weber, S. Pané and B. J. Nelson, Adv. Funct. Mater., 2018, 1804107, DOI: 10.1002/adfm.201804107.

39 M. K. Kuimova, Phys. Chem. Chem. Phys., 2012, 14, 12671-12686. 